\title{
Investigating Fit in Motion with a 4D Photogrammetry Scanner System
}

\author{
Anke KLPESER *, Angela MAHR-ERHARDT, Simone MORLOCK \\ Hohenstein Laboratories GmbH \& Co. KG, Germany
}

https://doi.org/10.15221/21.13

\begin{abstract}
Fit in motion is of vital importance for sports- and workwear. Ashdown et al. (2011) point out that valid fitting tests need to be performed in typical sports or work related movements. [1] Only when the garment fits well in specific positions it can protect the user without restriction of the range of motion. Therefore, a comprehensive knowledge about dynamic anthropometry is needed. Bye (2006) [2] and Bougourd (2014) [3] believe in this as an important research field within clothing technology. In the past sizing surveys issuing the change of body dimensions due to movement could only be performed with traditional anthropometric devices. This was time consuming and therefore often uncomfortable for the participants. Through 3D scanning technology sound implementation of big analysis in dynamic anthropometry are possible. [4] Consequently, several research projects were conducted investigating everyday, work and sport related static poses. Significant body surface and measurement changes due to movement were found. [5-7]

$3 \mathrm{D}$ scanning is not only a reliable method to capture body measurements but is utilized for garment fit investigations as well. [1, 8-11] So far, research was performed in regard of static poses. The further development from 3D to 4D changed the conditions. The interaction between garment und body in motion can be investigated with 4D scanning technology comprehensively.

In a basic research project, photogrammetry scanner "little Alice" was utilized for fit in motion assessment. "Little Alice" consists of 38 digital SLR cameras. Like every digital camera, it is possible to perform serial recording. This enables scanning in motion or 4D scanning. Three frames per second are recorded and moderate movements can be captured. In this study 25 male test subjects aged between 22-65 years ( $\varnothing 37.86 \pm 12$ ) with an average body height of $\varnothing 181.49 \mathrm{~cm}( \pm 6)$ and chest girths 96-120 cm (German sizes 48-60) participated. They were all either athletes or physical workers. Supporting companies provided work- and sportswear clothing systems (jackets and pants). Three movements were scanned with up to seven frames. Test subjects were scanned in static poses and dynamic sequences, both in underwear and in work- or sportswear. The reference scans were performed in Vitus Smart XXL system. In addition, each scan or sequence was captured twice.

4D scanning technology can be utilized to investigate fit in motion, assessment matrices were developed, air gaps and penetration areas were identified to compare different products. 4D scans are an excellent basis for comprehensive analysis of body-garment interaction. The limitation of the study is the focus on male subjects. More studies should be performed regarding female subjects, different movements and different garments.
\end{abstract}

Keywords: 4D bodyscanning, garments, fit, photogrammetry, fit in motion

\section{Introduction}

Garments interact with the wearer. On the one hand, they protect against cold, heat, rain or in the scope of personal protective equipment (PPE) even harmful substances. On the other hand, they restrict the wearer's movement. Multiple factors have an impact on garment user interaction regarding range of motion. These are textile properties, workmanship, pattern, size, fit and the users' bodily characteristics. [1] For sportswear knitted textiles are used to enable the wearer's mobility. Yet, textiles with special functionality as deployed in workwear or PPE are mostly rigid. Movement widths must be provided by pattern construction. The success of the ergonomic realization of garment pattern is assessed by fitting tests. Ashdown et al. (2011) point out that they need to be performed in typical sports or work related movements to be valid. [1] Only, when the garment fits well in specific positions it can protect the user without restriction of the range of motion. This underpins the importance of fit in motion for sports- and workwear.

Body measurements and geometry changes in movement. Therefore, a comprehensive knowledge about dynamic anthropometry is needed for ergonomic garment construction. Bye (2006) [2] and Bougourd (2014) [3] believe in this as an important research field within clothing technology.

\footnotetext{
*a.klepser@hohenstein.de; +49-7143-271325; www.hohenstein.de
} 
In the past, measurement tools for dynamic anthropometry were analog, e.g. measurement tape or caliper. [12] Thus, the investigation of alteration of the body was time-consuming and only possible at great effort. Through 3D scanning technology sound implementation of big analysis in dynamic anthropometry are possible. [4] So far, only static poses were analyzed in research projects. [4, 6, 13, 14] Three-dimensional capturing of human bodies is a stable and reliable method not only to investigate body changes. In addition, it can be used to analyze the fit of garments as well as the interaction of garment and wearer. [1, 8-11] Further development of scanning systems towards 4D or dynamic scanning enables comprehensive investigation in the area of dynamic anthropometry and garment fit in motion. Finally, dynamic movement and the resulting changes in body dimensions can be observed. Furthermore, the influence of garment on user movement can be examined.

4D scanner systems are more and more obtainable in the market. Common technologies are among others structural light and photogrammetry. [15-17] Manufacturers and developers are constantly working on 4D scanning systems. The competition leads to systems that are more sophisticated. Unfortunately, 4D scanners are expensive and require much space. Therefore, mostly big companies and research institutes utilize the new technology. Moreover, at the project starting point there were no research projects utilizing 4D scanner systems in the field of clothing technology to either analyze body measurement changes or fit in motion. It needs to be investigated and defined how to take body measurements from the moved body. New methods for fit assessment with a meaningful deployment of 4D data need to be developed. This is where the basic research project "Mobility restrictions" came in. Photogrammetry scanner "little Alice" was utilized for fit in motion assessment. The goal was to investigate the usability of the low-prized system for examination of garment wearer interaction. In addition, fit in motion testing workflow based on 4D scans was developed. Assessment matrices were created, air gaps and penetration areas were identified to compare different products.

\section{Method}

\subsection{Photogrammetry Scanner "little Alice"}

The scanner system "little Alice" is in its basic setup a 3D fullbody scanner to capture objects (human beings) with texture. It consists of 38 digital SLR cameras, six led panels and three flashlights. Four control computers are synchronizing each capture. The cameras were controlled with software CaptureGRID. [18] "Little Alice" is based on an ellipsoid, a three-dimensional ellipse (see Figure 1). Floor space is $3.3 \mathrm{~m} \times 3.2 \mathrm{~m}$ and height is $2.6 \mathrm{~m}$. The capturing area is $65.0 \times 64.0 \mathrm{~cm}$. Digital SLR cameras provide single and serial capturing. Therefore, $4 \mathrm{D}$ scanning is possible. Each capture or frame includes 38 pictures. Three frames per second can be performed with a maximum of seven frames in total. To calculate the 3D scan, software RealityCapture was used. [19]

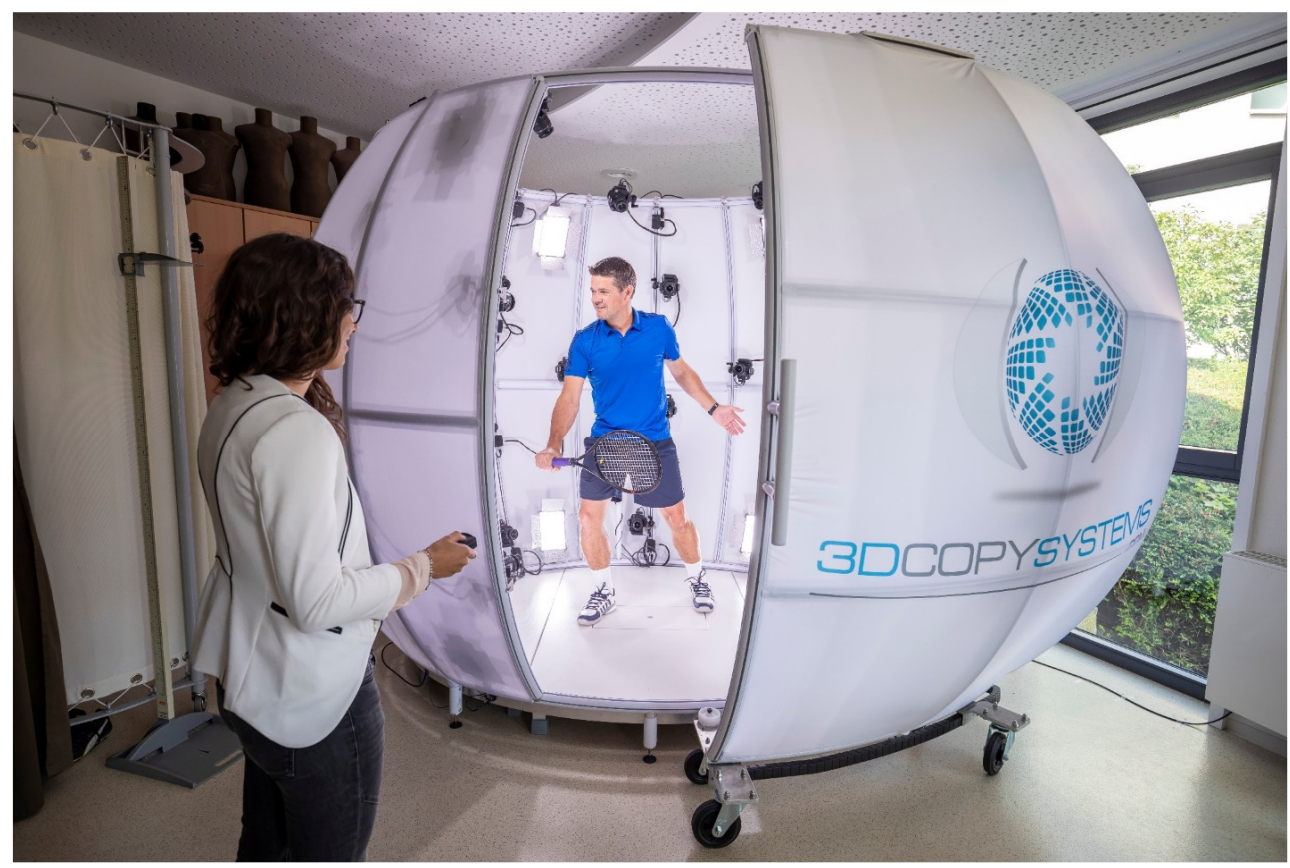

Fig. 1. Photogrammetry scanner "little Alice". 


\subsection{Captured Movements}

In the study three work or sport related movements were defined. Primary aim was that each movement should lead to distinct differences in body measurements. Each motion was captured in underwear and work- or sportswear. For the fit in motion analysis two movements were of importance, "Arm Flex" and "Leg Flex". They were defined by three parameters: Starting position, motion sequence and end position. "Arm Flex" leads to an elongation of the back width and arm reach in mean of $31.5 \%$. Therefore, it was taken for the investigation of jacket's fit in motion (see Table 1 and Figure 2). "Leg Flex" was chosen to investigate the fit of pants (see Table 1 and Figure 3). The most important position in this sequence is when the test subject steps on the ladder. Hip and knee joint show a 90-degree angle and therefore lead to an increase of length at the backside of the waist hip area and an increase of the leg length measured ventral on the leg front side. Knee flex leads to an elongation in mean of $11 \%$ in leg length.

Table 1. Description of captured motions.

\begin{tabular}{|c|c|c|c|}
\hline & Starting position & Motion sequence & End position \\
\hline Arm Flex & $\begin{array}{l}\text { Legs hip width apart, arms on } \\
\text { the side }\end{array}$ & $\begin{array}{l}\text { Arms moved stretched on } \\
\text { frontal plan to shoulder level, } \\
\text { on transversal plan to front of } \\
\text { the body }\end{array}$ & $\begin{array}{l}\text { Legs hip width apart, arms } \\
\text { stretched on shoulder level in } \\
\text { front of the body }\end{array}$ \\
\hline Leg Flex & $\begin{array}{l}\text { Legs hip width apart, arms in } \\
\text { front of the body, hands on } \\
\text { the ladder }\end{array}$ & $\begin{array}{l}\text { Lifting right leg until } 90 \\
\text { degrees hip and knee flexion; } \\
\text { right food is placed on second } \\
\text { step, left food follows }\end{array}$ & $\begin{array}{l}\text { Both feet are placed on } \\
\text { second step, legs stretched, } \\
\text { hands on ladder }\end{array}$ \\
\hline
\end{tabular}

Starting position

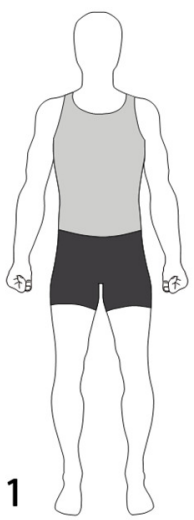

Motion sequence

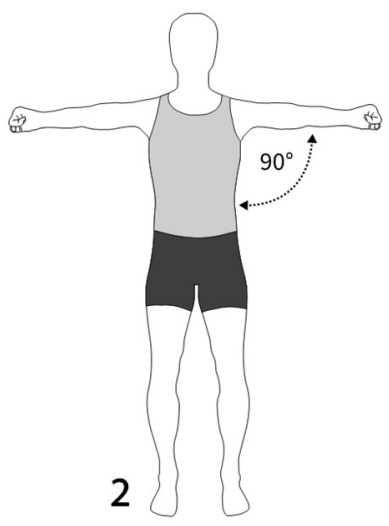

End position

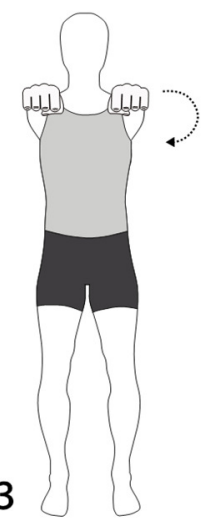

Fig. 2. Sketch: Captured movement "Arm Flex".

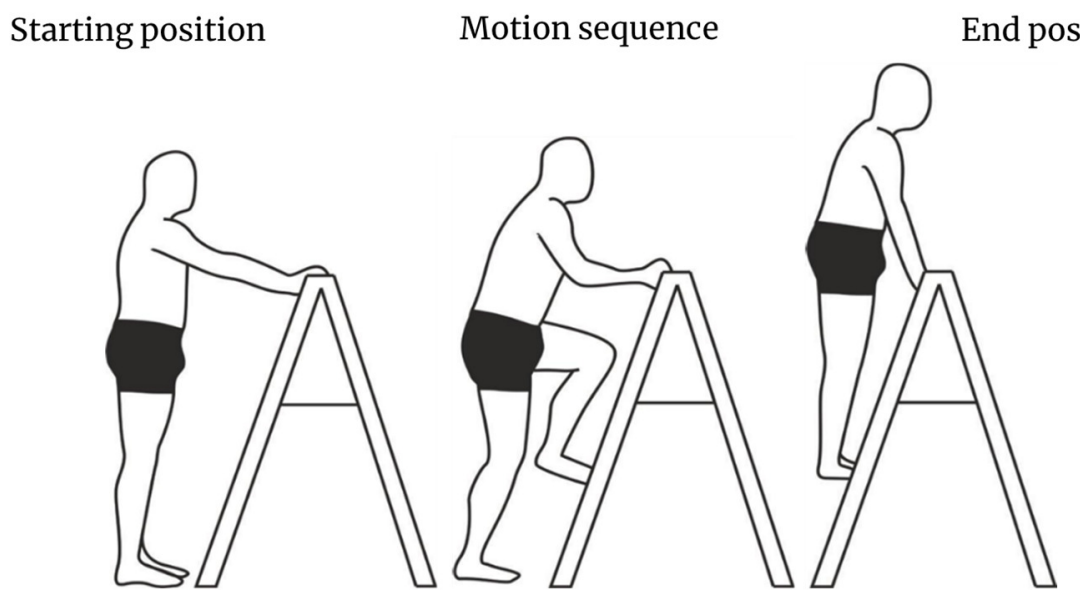

Fig. 3. Sketch: Captured movement "Leg Flex".

Two reference scans were performed with Vitus Smart XXL scanner system. Each scan or scan sequence was captured twice. 


\subsection{Sample}

25 male test subjects aged between 22 and 65 years $(\varnothing 37.86$ Jahre $( \pm 12))$ participated in the study. They were either performing sports regularly or worked physically. It was assumed, that body measurement changes will be more distinct within this target group. It was a random selection. The average body height was $181.49 \mathrm{~cm}( \pm 6)$. The subjects had chest girths between 96.0 and $120.0 \mathrm{~cm}$, this corresponds with German sizes 48-60.

Supporting companies provided work- and sportswear clothing systems (jackets and pants) in the sizes 50, 54 and 58. Therefore, some test subjects had to wear clothing not their size. It was assumed that the fit in motion effects would again be more distinct, if a person wears products to small.

In total more than 4000 scans/frames were calculated, 164 scans per person.

\section{Results}

In movement garment fit problems may occur. These manifest themselves in aesthetic issues and functional limitations. Sports- and workwear is supposed to not restrict the wearer, to cover the body even in dynamic movement and to protect against external influences. To assess the fit in motion, workflows based on 4D scans were developed. Distance analysis were performed and assessment matrices created. Garment wearer interaction was analyzed and areas with air gaps and penetration identified. Therefore, each test subject was scanned in underwear and in garment systems.

\subsection{Distance Analysis}

Body measurements and geometry are changing while the body moves. Due to the alteration of the body surface garments are repositioned. For example, sleeves and trouser legs slip towards the body center. Based on the 4D scan data distance analysis between landmarks on the body and on the garment seams (waistband, trouser leg seam and sleeve seam) were performed.

The repositioning of the waistband was analyzed with scans of the "Leg Flex" movement. A marker was placed on the waist point (waist level on spine) to assess the distance to the waistband in the starting position and with 90-degree angles in hip and knee joint (see Figure 4).
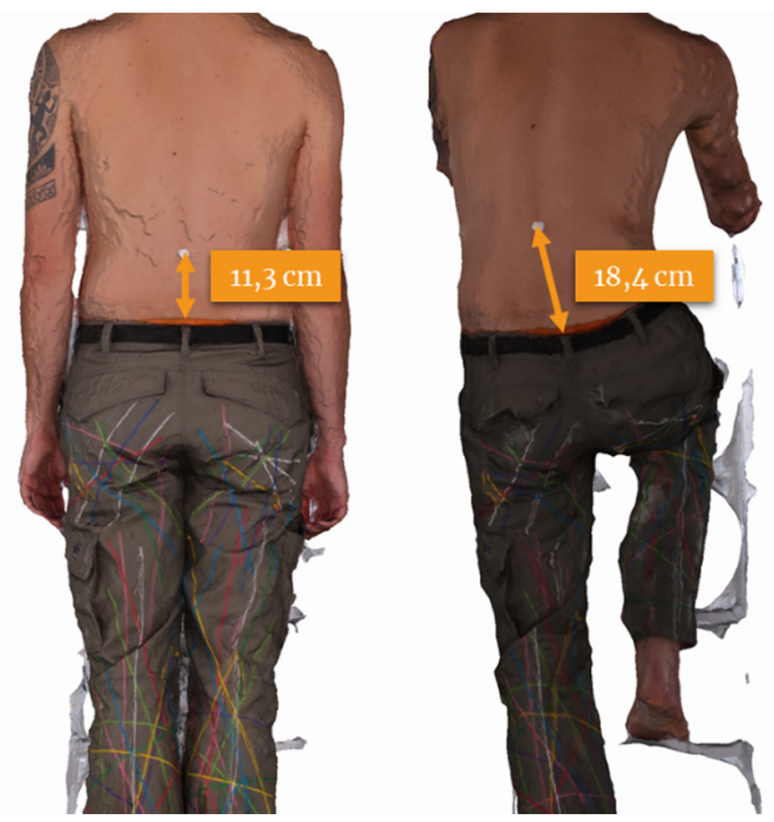

Fig. 4. Distance analysis waist point to waistband.

In mean, the distance was $10.3 \mathrm{~cm}$ in the starting position. In position 2 (90 degree in hip and knee joint) the mean distance was $16.3 \mathrm{~cm}$. This is a percentage deviation of $36.5 \%$. Based on these findings an assessment system for classification was developed (see Table 2). The five categories are similar to school grading systems, starting from a percentage less than 19.9 for an A as excellent and ending at a percentage more than 50.0 for an $\mathrm{E}$ as a fail 
Table 2. Assessment matrix distance waist point to waist band.

\begin{tabular}{|c|c|}
\hline Percentage deviation & Assessment \\
\hline$<19.9 \%$ & $\mathrm{~A}$ \\
\hline $20.0-29.9 \%$ & $\mathrm{~B}$ \\
\hline $30.0-39.9 \%$ & $\mathrm{C}$ \\
\hline $40.0-49.9 \%$ & $\mathrm{D}$ \\
\hline$>50.0 \%$ & $\mathrm{E}$ \\
\hline
\end{tabular}

\subsection{Garment Wearer Interaction Analysis}

Basis for the garment wearer interaction analysis were captured movements in underwear and in the garment systems. The scans in underwear show the space that the subjects body geometry would occupy unaffectedly. The scans in garment show the body in possible restricted condition. Both scans were merged to investigate the body garment interface. For the scan processing, software Geomagic Studio was utilized. [20] The scans were imported, aligned, and colored or set transparent. The analyzing method enables a visualization of air gaps and penetration areas. Air gaps are areas where the garment shows great distance to the body. Penetration areas restrict the space occupation of the body.

The garment wearer interaction analysis was performed with jackets and pants (see Figure 5 and Figure 6 ). Investigating the provided jackets, revealed a penetration area lateral at the torso. $70 \%$ of all merged scans showed the musculus latissimus dorsi of the scan in underwear, a muscle at the back and side of the torso, penetrating the scan with garment (see Figure 5, no 1). This muscle needs more space to perform the "Arm Flex" movement. This indicates that the jackets may restrict the wearer in this motion. In addition, on $80 \%$ of the test subjects there was a small penetration at the side of the shoulder. In parts big air gaps occurred at the front side of the torso. This was determined in $90 \%$ of the test subjects. In $30 \%$ of the examined cases, the jacket was repositioned upwards due to the arm movement on shoulder level. Body areas on the front side of the torso were sometimes uncovered (see Figure 5, no 3).

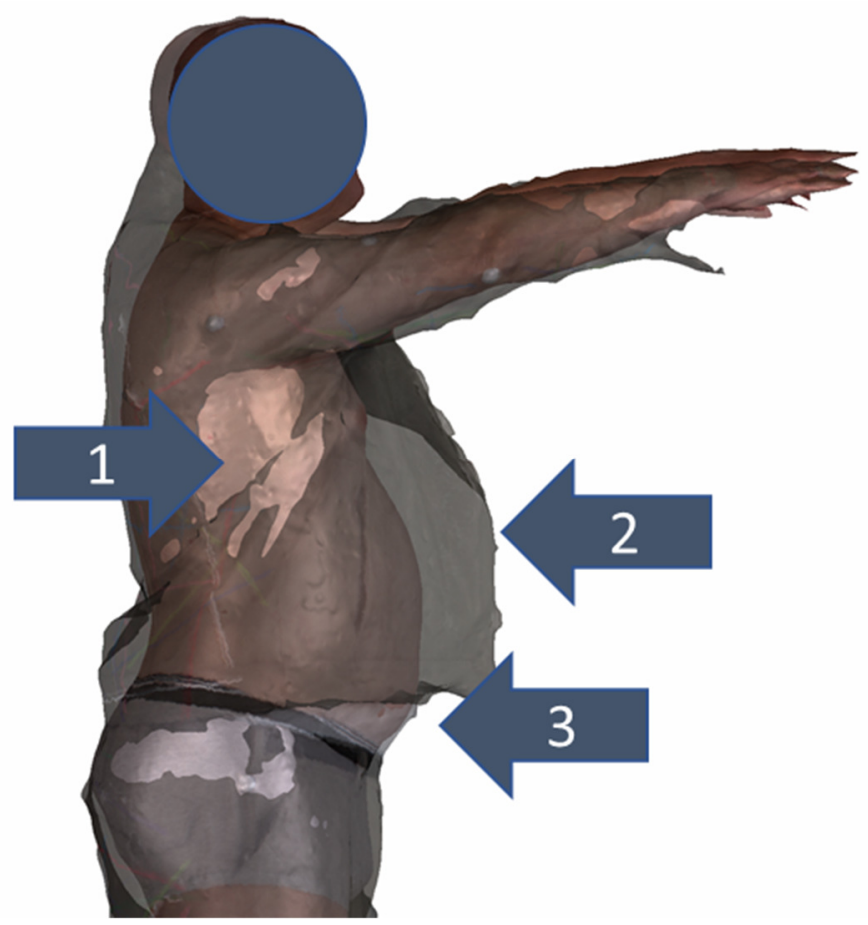

Fig. 5. Work wear jacket: Air gaps, penetration, and uncovered body areas.

The Investigation of the provided pants revealed in $87,5 \%$ a penetration of the left back trouser leg (see Figure 6, no 1). In $75 \%$ there was a penetration area at the right leg on the front part of the pants (see Figure 6, no 2). Both areas visualize the tension in the product caused by the movement "Leg Flex". Garment pressure can occur on the back part of the left leg and the front part of the right leg. Therefore, movement can be restricted. Finally, $100 \%$ of the scans showed a contact area at the buttock area (see Figure 6, no 3). 


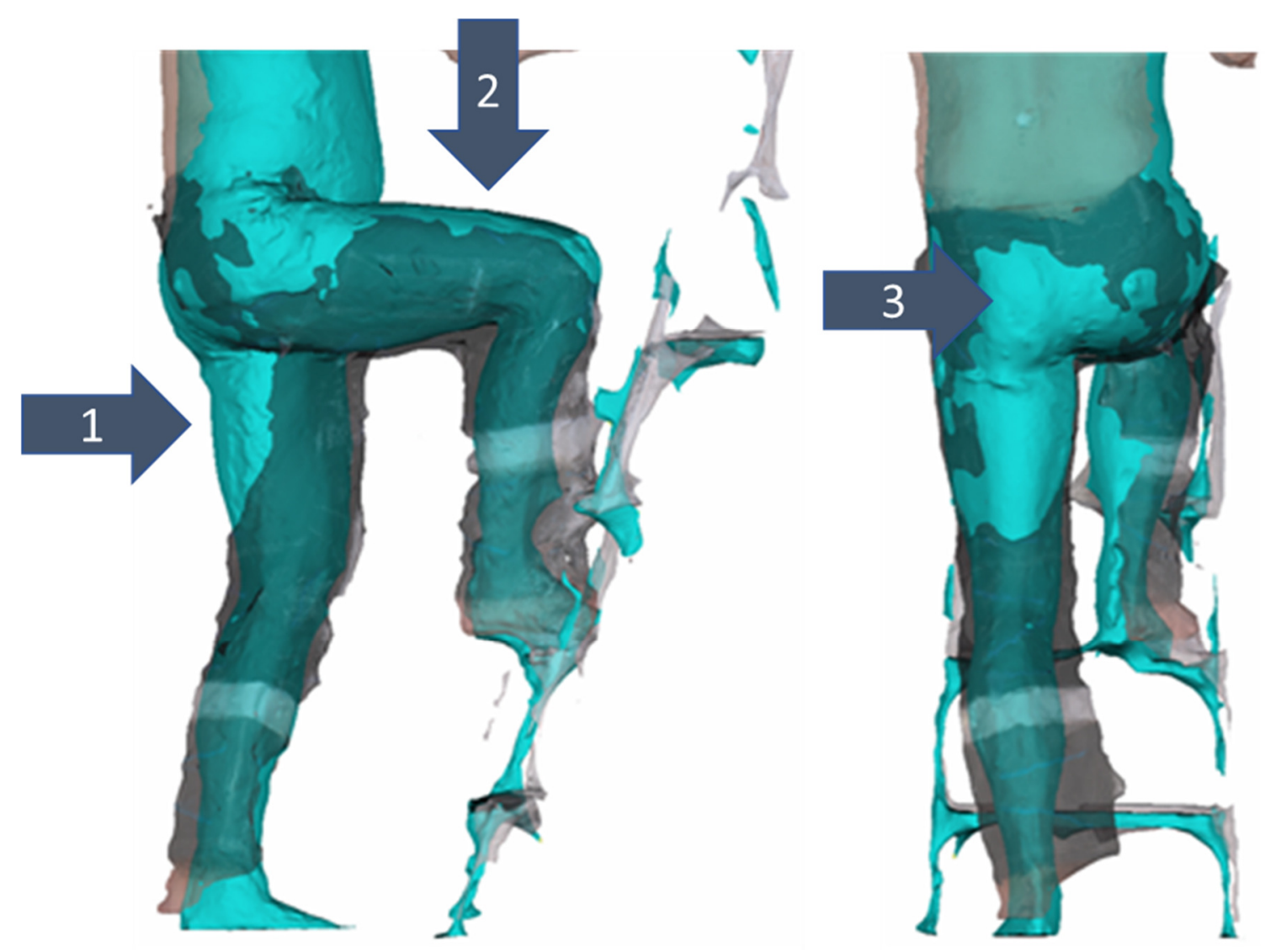

Fig. 6. Work wear pants: Air gaps, penetration, and uncovered body areas.

\section{Conclusion}

Garment and especially sports and work wear are used not only in static pose but first of all in movement. The special functionality must be provided in motion. Yet, the protection against external influences and ergonomic fit are not compatible in all cases. Often high performance personal protective equipment restricts the range of motion. Multiple factors influence mobility restrictions. Therefore, the development of ergonomic and functional garment is a big challenge. The knowledge of the garment wearer interaction is of great importance.

So far, traditional fitting test are performed without three-dimensional scanner technology. Yet, 3D and even more 4D scans are an excellent basis for comprehensive analysis in fit in motion. The scans visualize the garment fit in a whole movement sequence. Each position can be assessed by rotation and zooming the test person's scan. Different product versions can be compared or analyzed in various sizes. Merging scans in underwear and garments enables the investigation of air gaps and penetration areas. The distance analyzes with the evaluation matrices developed in this project provide an objective method to categorize garment performance in movement. 3D / 4D photogrammetry scanner "little Alice" is a useful and low-cost tool for fit in motion testing. Yet, the 38 cameras are the bottom line for fullbody scans. Like with every scanner system only areas that are seen by the cameras or sensors can be captured. More cameras as provided in the "big Alice" system will lead to an optimized coverage. Yet, this will naturally raise the costs.

Providing the basis for the merging of two scans in the same posture is a major challenge in scanning in motion. Instructing test persons to perform defined poses in the scanner is complicated at any time. The position needs to be defined precisely and the test person needs to be able to follow the instructions. This is even more complex with whole movements. Therefore, the defined motions should be as simple and reduced to the most important body poses as possible. Furthermore, since "little Alice" captures only 3 frames per second, additional runs of the scanning process are necessary. Thus, the correct pose can be extracted and compared. 
Beside the challenges, fit in motion with 4D scanner systems should be further executed and investigated. More garments should be assessed, and garment wearer situation of females should be examined additionally. Further movements should be investigated as well. Moreover, 3D / 4D data can be used to validate 3D simulation output. The software in the market still needs improvement regarding avatar geometry in movement, material properties and garment wearer interaction. Future research is needed to optimize these tools for visualization and fit testing. It can be expected that 3D simulation systems will be the future in garment development, visualization and fitting. All the more, the available software should be validated and improved. Users must know how reliable the output of the digital fitting tests are.

\section{Acknowledgment}

The IGF project $20163 \mathrm{~N}$ by the research association Forschungskuratorium Textil e.V., Reinhardtstraße 12-14, 10117 Berlin, is financed through the AiF within the framework of the program for promotion of cooperative industrial research (IGF) by the German Federal Ministry for Economic Affairs and Energy based on a resolution by the German Bundestag.

\section{References}

[1] S. P. Ashdown, "Improving body movement comfort in apparel," in Improving comfort in clothing, G. Song, Ed., ed Cambridge (GB): Woodhead Publishing, 2011, pp. 278-302.

[2] E. Bye, K. L. Labat, and M. R. Delong, "Analysis of Body Measurement Systems for Apparel," Clothing and Textiles Research Journal, vol. 24, pp. 66-79, 2006, https://doi.org/10.1177/0887302x0602400202.

[3] J. Bougourd and P. Treleaven, "National size and shape surveys for apparel design," in Anthropometry, apparel sizing and design. vol. 148, D. Gupta and N. Zakaria, Eds., ed Cambridge (GB): Woodhead Publishing, 2014, pp. 141-166.

[4] S. Choi and S. P. Ashdown, "3D body scan analysis of dimensional change in lower body measurements for active body positions," Textile Research Jou., vol. 81, pp. 81-93, 2011, https://doi.org/10.1177/0040517510377822.

[5] I. Dabolina, E. Lapkovska, and A. Vilumsone, "Dynamic Anthropometry for Investigation of Body Movement Comfort in Protective Jacket," in Functional Textiles and Clothing, Singapore, 2019, pp. 241-259.

[6] S. Morlock, C. Loercher, A. Schenk, and A. Klepser, "Functional Body Measurements - MotionOriented 3D Analysis of Body Measurements," presented at the 3DBODY.TECH 2019 - 10th International Conference and Exhibition on 3D Body Scanning and Processing Technologies, Lugano (CH), 2019, https://doi.org/10.15221/19.244.

[7] L. Chi and R. Kennon, "Body scanning of dynamic posture," International Journal of Clothing Science and Technology, vol. 18, pp. 166-178, 2006, https://doi.org/10.1108/09556220610657934.

[8] F. Baytar, J. Aultman, and J. Han, "3D Body Scanning for Examining Active Body Positions: An Exploratory Study for Re-Designing Scrubs," presented at the 3rd International Conference on 3D Body Scanning Technologies, Lugano (CH), 2012.

[9] J. Nam, D. H. Barnson, S. P. Asdown, H. Cao, and B. P. Jin, S., "Fit Analysis of Liquid Cooled Vest Prototypes Using 3D Body Scanning Technology," Journal of Textile and Apparel, Technology and Management, vol. 4, pp. 1-15, 2005.

[10] N. Nawaz, O. Troynikov, and K. Kennedy, "Investigation into Fit, Distribution and Size of Air Gaps in Fire-Fighter Jackets to Female Body Form," presented at the 3rd International Conference on 3D Body Scanning Technologies, Lugano (CH), 2012.

[11] S. Morlock, "3D-basierte Entwicklung eines innovatven Verfahrens zur Passformdiagnose von Bekleidung " Hohenstein Institut für Textilinnovation gGmbH2016.

[12] U. Schmid and J. Mecheels, "Kräfte an Textilien und Nähten der Kleidung in Abhängigkeit von Körperbewegungen und Kleidungsschnitt," Bekleidung \& Wäsche, vol. 2, pp. 77-82, 1981.

[13] S.-Y. Choi and S. P. Ashdown, "Application of Lower Body Girth Change Analysis Using 3D Body Scanning to Pants Patterns," Journal of the Korean Society of Clothing and Textiles, vol. 34, pp. 955-968, 2010. 
[14] J. Lee and S. P. Ashdown, "Upper Body Surface Change Analysis using 3-D Body Scanner," Journal of Korean Society of Clothing and Textiles, vol. 29, pp. 1595-1607, 2005.

[15] 3dMD. (26.08.2021). Temporal-3dMD Systems (4D). Available: https://3dmd.com/products/

[16] Instituto de Biomecanica de Valencia (IBV). (26.08.2021). Move 4D. Available: https://www.ibv.org/tecnologias/analisis-de-movimientos-4d/move-4d-3/

[17] 3DCOPYSYSTEMS. (26.08.2021). 3DCOPYSYSTEMS. Available: https://3dcopysystems.com/

[18] CaptureGRID. (26.08.2021). CaptureGRID. Available: https://capturegrid.com/

[19] CapturingReality. (26.08.2021). RealityCapture. Available: https://www.capturingreality.com/

[20] 3D Systems Inc. (30.08.2021). Geomagic Studio 2012. Available: https://de.3dsystems.com/software 